\title{
Effects of antipsychotic medication on electromyographic responses to transcranial magnetic stimulation of the motor cortex in schizophrenia
}

\author{
Nicholas J Davey, Basant K Puri, Helen S Lewis, Shon W Lewis, Peter H Ellaway
}

Department of Physiology, Charing Cross and Westminster Medical School, University of London N J Davey

H S Lewis

P H Ellaway

Robert Steiner Magnetic Resonance Unit, Royal

Postgraduate Medical School, Hammersmith Hospital, London B K Puri

Department of Psychiatry, University of Manchester,

England

$\mathrm{S} W$ Lewis

Correspondence to: Dr NJ Davey, Department of Physiology, Charing Cross and Westminster Medical School, St Dunstan's Road, London W6 8RP, UK.

Received 30 October 1996 and in revised form 8 April 1997

Accepted 24 April 1997

\begin{abstract}
Objective-To assess the effect of antidopaminergic antipsychotic medication on the electromyographic (EMG) responses of thenar muscles to transcranial magnetic stimulation (TMS) of the motor cortex in schizophrenic patients.

Methods-A group of nine drug naïve schizophrenic patients was compared with a group of nine schizophrenic patients established on neuroleptic medication. Surface EMG recordings were made from the thenar muscles while patients maintained a weak isometric voluntary contraction. TMS was applied using a $9 \mathrm{~cm}$ circular stimulating coil centred over the vertex. The EMG responses to up to 50 magnetic stimuli were rectified and averaged.
\end{abstract}

Results-There was no difference in threshold TMS strength for eliciting compound motor evoked potentials (cMEPs), or in their latency, in drug naïve and medicated patients. In some patients the silent period (SP) was clearly made up of two parts and the percentage of control levels of voluntary EMG was measured in each component. During the early component of the SP there was a weaker $(P<0.05)$ suppression of EMG in the medicated patients (mean 73.9 (SEM) $5.5 \%$ of control levels) compared with the drug naive patients (54.7 (SEM) $7.3 \%$ of control levels). This resulted in the latency of maximum suppression of voluntary EMG being longer $(P<0.05)$ in the medicated patients (38.3 (2.4) $\mathrm{ms}$ ) than in the drug naïve patients $(28.2(0.7) \mathrm{ms})$. During the late component of the SP voluntary EMG was reduced to similar levels $(P>0.05)$ in both medicated $(48.2$ (7.7)\% of control levels) and drug naïve (58 $(7.8) \%$ of control levels) patients.

Conclusion-The results are discussed with reference to the disrupted inhibition seen in the early part of the SP in Parkinson's disease and drug induced parkinsonism. The future uses of motor responses to TMS as a marker for the status of antipsychotic medication are considered.

\section{(F Neurol Neurosurg Psychiatry 1997;63:468-473)}

Keywords: antipsychotic drugs; schizophrenia; transcranial magnetic stimulation
Since the report by Carlsson and Lindquist ${ }^{1}$ that the typical antipsychotic drugs chlorpromazine and haloperidol give rise to an accumulation of dopamine metabolites in dopamine rich brain areas in mice, it has been found that all effective antipsychotic drugs bind to dopamine receptors. The degree of dopamine D2 receptor binding correlates with the clinical effectiveness of typical antipsychotic drugs. ${ }^{2}{ }^{3}$ Furthermore, there are delayed actions of these drugs on presynaptic dopamine activity that closely parallel the time course of their therapeutic action. ${ }^{4}$ The mode of action of this class of drugs remains uncertain, and more recent studies (see review $^{5}$ ) have implicated several other neurotransmitters and neurohormones.

Transcranial magnetic stimulation (TMS) using a coil delivering a rapidly changing magnetic field ${ }^{6}$ is a method that can be used safely and painlessly to stimulate the motor cortex through the intact skull. When stimulation is delivered using a circular coil centred over the vertex, compound motor evoked potentials (cMEPs) are produced in skeletal muscles served by the motor cortical region lying beneath the coil. The cMEPs are produced at lowest stimulus threshold when the current induced in the brain flows in an anteromedial direction through the relevant part of the primary motor cortex. ${ }^{7}$ When the subject makes a voluntary contraction in a muscle, facilitation occurs in the corticospinal pathway resulting in an increase in amplitude (and decrease in stimulus threshold) of the cMEPs. ${ }^{8}$ In a previous study ${ }^{9}$ we found that cMEP latency was reduced in drug naïve schizophrenic patients compared with normal control subjects; there was no difference in the silent period of EMG after the cMEP. We have now investigated the effects of antipsychotic medication on characteristics of the cMEPs and the subsequent silent period in drug naïve schizophrenic patients.

Preliminary reports of this study have been published in abstract form. ${ }^{10} 11$

\section{Methods}

SELECTION OF PATIENTS

A group of nine neuroleptic drug free schizophrenic patients (seven men, two women; ages 30-45 years) were compared with a group of nine schizophrenic patients (seven men, two women; ages 29-42 years) established on 
antipsychotic medication. All the patients met DSM-IV criteria for schizophrenia. ${ }^{12}$ Seven of the neuroleptic drug free patients were antipsychotic naïve and the remaining two had not received antipsychotic medication for at least six months. Andreasan's rating scales were used to assess the predominance of positive (SAPS) and negative (SANS) symptoms in drug naïve and medicated patients. The mean (SD) SAPS score was 35.2 (15.9) in the medicated patients and $41.0(22.2)$ in the drug naive patients. The mean SANS (SD) score was 15.0 (16.9) in the medicated patients and 17.6 (15.7) in the drug naïve patients. There was no significant difference (independent $t$ test; $P>0.05)$ for either score between the two groups of patients. There was no difference (Mann-Whitney rank sum test; $\mathrm{P}>0.05$ ) in the mean (SD) duration of the illness between the two groups (medicated 25.9 (27.9) months; drug naïve 30.8 (27.6) months).

The patients receiving antipsychotic medication were taking an average (SEM) daily chlorpromazine equivalent dose of 528 (166) mg (range $200-1800 \mathrm{mg}$ /day). Clinical assessments of the patients were made using the clinical global impression (CGI) scale ${ }^{13}$ ranging from a score of 1 "being normal" to a score of 7 "being among the most extremely ill patients". The clinical status of patients in the group taking antipsychotic medication had shown an improvement of at least 2 points on the CGI scale after commencement of medication before physiological recordings were made; such clinical improvement took at least two weeks to occur. Three of the patients were examined both before and after starting antipsychotic medication. Ethical approval for this investigation was obtained from Charing Cross and Westminster Medical School, and all subjects gave informed consent to take part in the study.

ELECTROMYOGRAPHIC (EMG) RECORDINGS Electromyograms were recorded from the dominant hand using two self adhesive surface electrodes (Arbo Neonatal Pink). One electrode was placed on the thenar eminence and the other on the dorsal aspect of the interphalangeal joint of the thumb. A metal plate was strapped to the wrist to act as an earth electrode. All EMG signals were filtered $(-3 \mathrm{~dB}$ at $100 \mathrm{~Hz}$ and $2 \mathrm{kHz}$ ) and differentially amplified $(\times 1000)$ before being sampled (digitisation at $4 \mathrm{kHz}$ ) by a computer for analysis (Cambridge Electronic Design 1401/IBMcompatible PC) and recorded on a magnetic digital audiotape (DAT) recorder (Teac, RD130T PCM Data Recorder). The EMG records were full wave rectified and averaged with reference to the stimulus using a signal analysis program (SIGAVG, Cambridge Electronic Design).

TRANSCRANIAL MAGNETIC STIMULATION (TMS) Electromagnetic stimulation of the cerebral motor cortex was achieved using a Magstim Model 200 stimulator (The Magstim Company Limited, UK). This was connected to a circular (9 cm average diameter; 14 turns) stimulating coil with a peak electric field strength of $530 \mathrm{~V} / \mathrm{m}$ and a peak magnetic field strength of 2.0 Tesla. Cross wires were taped to either side of the coil to assist in its accurate placement. To elicit responses from the right thenar muscle, the coil was placed tangentially on the head with its centre over the vertex. The initial current flow in the coil was in an anticlockwise direction when viewed from above. ${ }^{14}$

\section{EXPERIMENTAL PROCEDURE}

Subjects were seated comfortably with their arms passively supported. They were instructed to adduct the thumb against the forefinger with a mild pressure sufficient to evoke a background EMG. Audio feedback of EMG signals through a loudspeaker and a visual display by means of a cathode ray oscilloscope were provided to enable subjects to maintain a steady pressure of 5\%-10\% maximum voluntary contraction in the thenar muscles during the recordings. The stimulus strength was increased in increments of $2 \%$ of maximum stimulator output from just below threshold for any response to a strength 5\%-10\% maximum stimulator output above threshold for a compound motor evoked potential (cMEP). If no response was seen after 50 stimulus presentations, the stimulus strength was deemed to be below threshold.

\section{MEASUREMENT AND ANALYSIS OF RESULTS}

Measurements of latency of cMEP, latency of maximum suppression, and amount of suppression during the early and late parts of the silent period were made from records produced at stimulus intensities $2 \%-5 \%$ maximum stimulator output above threshold for evoking cMEP responses. Averaged rectified and unrectified records were used together to increase our confidence that the measurements were accurate. The time when reduction of EMG became maximum during the silent period was not always clear due to the noise level of the EMG signal. For this reason, the cumulative sum (cusums) derivatives ${ }^{15}$ of the digitised rectified averages were examined; this process was carried out independently by two people.

The extent of the reduction of voluntary EMG during the silent period was assessed from the averaged, rectified records as follows (fig 1):

(1) Each record was inspected to ascertain whether the silent period consisted of an immediate drop in EMG to the maximum level of suppressed activity or whether the maximum level of suppression occurred with a distinct delay. (2) In patients where two components were present, cursors were set at positions equivalent to the start and finish of the first component (fig 1, cursors 3 and 4). The mean amplitude of EMG between the cursors was assessed, this was termed the "early SP". In patients where two components were not identifiable visually, the silent period was divided into two equal halves. (3) The mean amplitude of EMG was assessed during an equivalent duration later portion of the silent period starting at the onset of maximum suppression (fig 1 , 


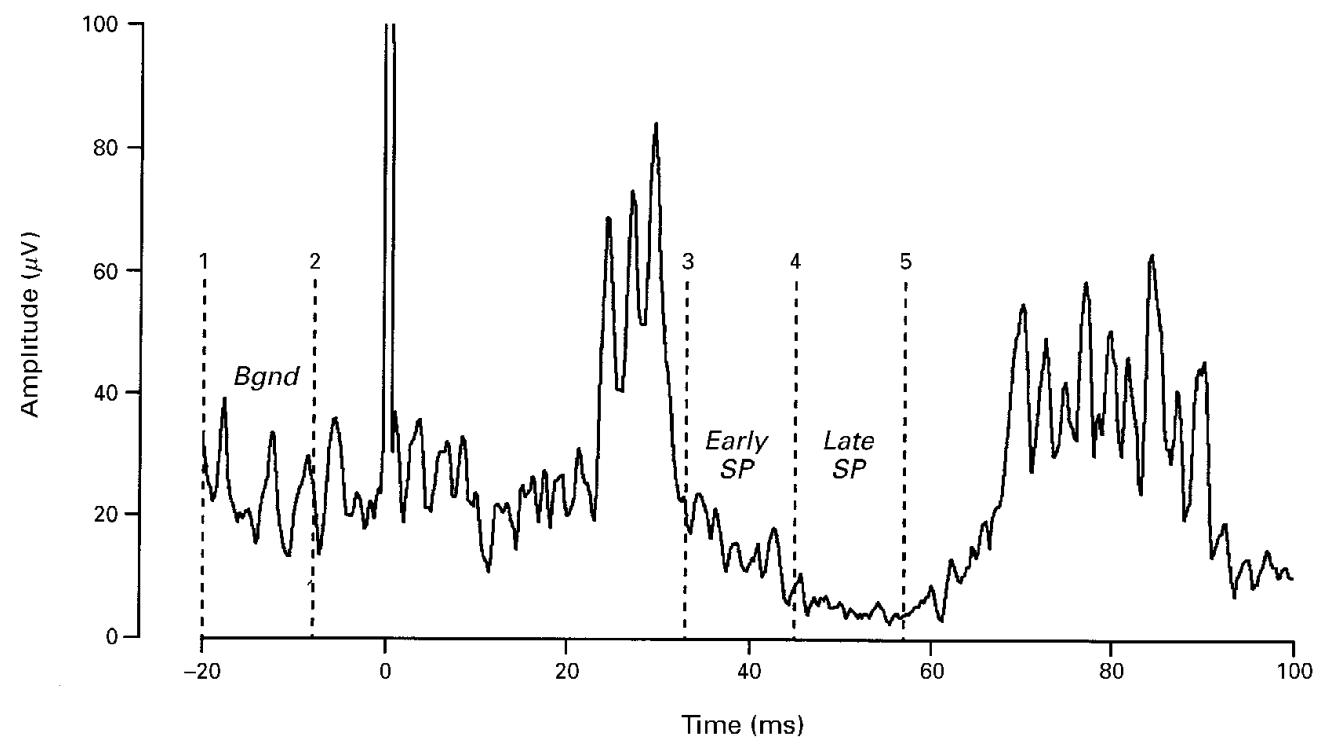

Figure 1 Averaged rectified motor response to TMS during weak voluntary contraction of thenar muscles in a medicated patient. Stimulus strength $34 \%$ MSO, average of 20 responses. The silent period has an early component (early SP) delineated by cursors 3 and 4 and lasting $12 \mathrm{~ms}$, before suppression of voluntary EMG becomes maximal. A later component (late SP), also of $12 \mathrm{~ms}$ duration is delineated by cursors 4 and 5 and a prestimulus portion of background EMG (bgnd), of $12 \mathrm{~ms}$ duration, is delineated by cursors 1 and 2 . The EMG is reduced to $60 \%$ of background levels during the early SP and to $25 \%$ of background during the late SP.
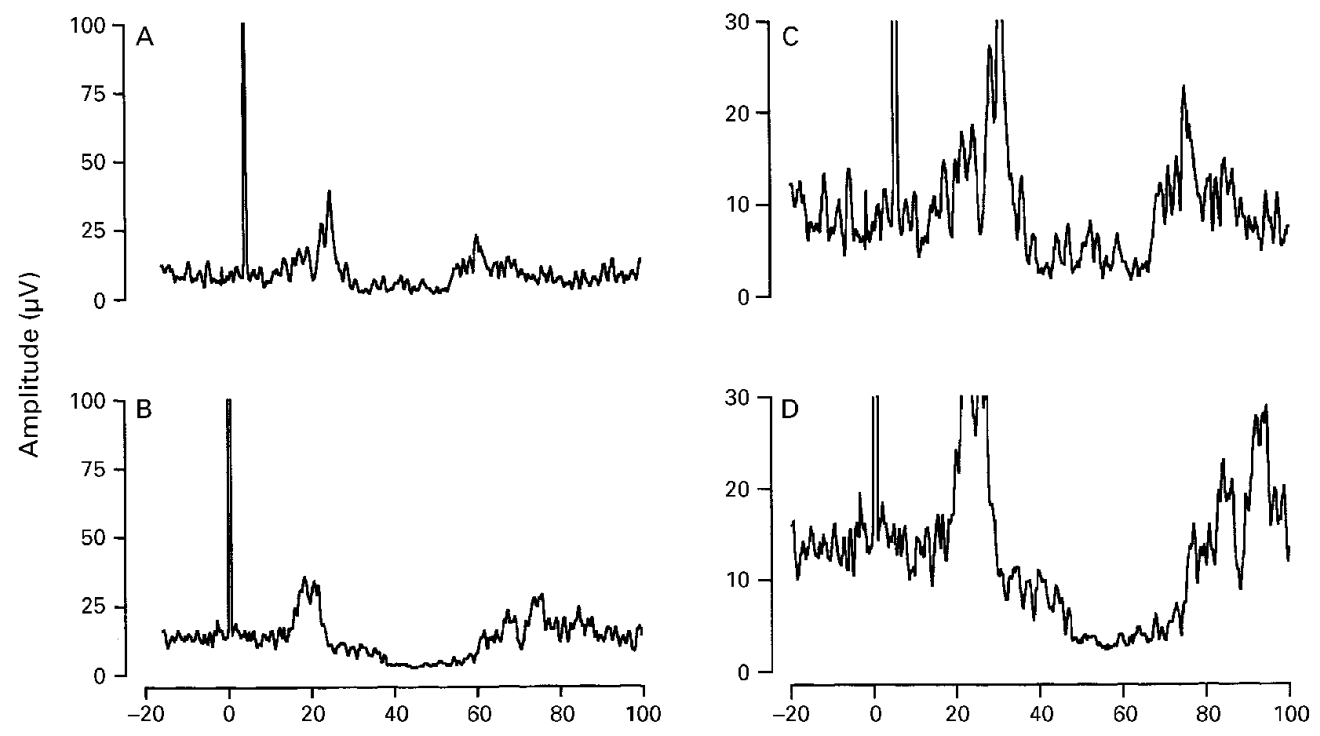

Time (ms)

Figure 2 Averaged rectified motor responses to TMS during weak voluntary contraction of thenar muscles in a drug naïve schizophrenic patient $(A, C)$ and a patient established on antipsychotic medication $(B, D)$. Records $C$ and $D$ are the same as $A$ and $B$ but have a higher amplification to allow better resolution of the silent period. Note the abrupt onset of the silent period in the drug naïve patient with suppression of EMG becoming maximal at a latency of $27 \mathrm{~ms}$. In the treated patient EMG is present after the cMEP for a period of $12 \mathrm{~ms}$ before maximum suppression develops, 40 ms after the stimulus. Average of 25 responses $(A, C)$ and 20 responses $(B, D)$. Stimulus strength $29 \%$ maximum stimulator output $(A, C)$ and $40 \%$ maximum stimulator output $(B, D)$.

cursors 4 and 5). This was termed the "late SP". (4) A control mean EMG level was measured in a further equivalent duration portion of the record before the time of stimulation (fig 1 , cursors 1 and 2). This was taken to represent the background level of EMG in the record and is termed the "bgnd". (5) The mean levels of EMG in early SP and late SP were expressed as percentages of the background level.

\section{Results}

Figure 2 shows typical responses to TMS in both a drug naïve patient (A and $\mathrm{C}$ ) and a patient several weeks after starting to take antipsychotic medication (B and D).

The cMEP evident in the drug naïve patient (fig1 A) has a similar latency $(20 \mathrm{~ms})$ to the cMEP present in the treated patient $(18.5 \mathrm{~ms})$ (fig1 B). Figure 3A shows the the bar chart of mean latencies of cMEPs for all subjects. The mean (SEM) latency of cMEPs did not differ significantly (unpaired $t$ test; $\mathrm{P}>0.05$ ) between drug naïve $(21.2(0.5) \mathrm{ms})$ and treated schizophrenic patients $(21.1(0.5) \mathrm{ms})$. The mean threshold for evoking cMEPs was no different (unpaired $t$ test; $\mathrm{P}>0.05$ ) in drug naïve patients 

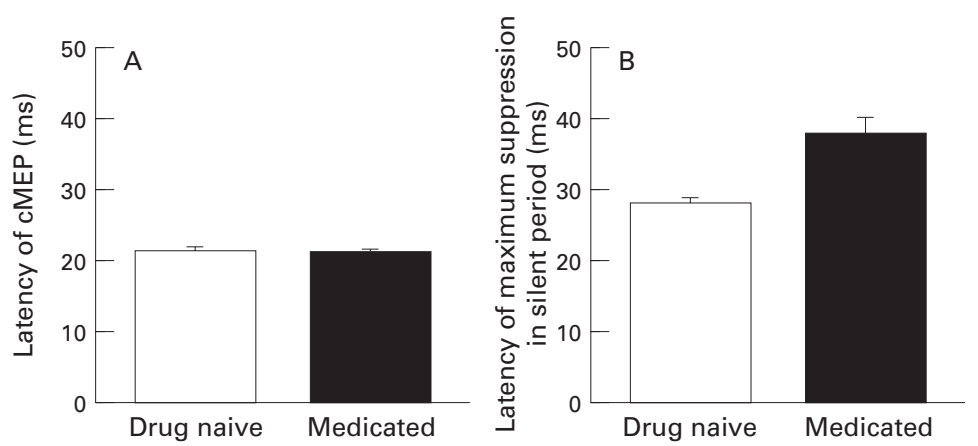

Figure 3 (A) Latency of cMEP responses measured in drug naïve patients $(n=9)$ and patients established on antipsychotic medication $(n=9)$. There is no statistical difference $(P>0.05)$ between drug naive and medicated patients. (B) Latency of maximum suppression of voluntary EMG measured in drug naïve patients $(n=9)$ and patients established on antipsychotic medication $(n=9)$. The latency of maximum suppression is longer $(P<0.01)$ in the treated patients. Error bars represent 1 SEM.

(27.9 (2.4)\% maximum stimulator output) and patients established on medication (32 (2.2)\% maximum stimulator output).

There was an abrupt onset of maximum EMG suppression in the silent period of the drug naïve patient (fig $1 \mathrm{~A}, \mathrm{C}$ ) compared with a silent period consisting of two discrete components in the treated patient (fig $1 \mathrm{~B}, \mathrm{D}$ ). Examination of the records from the treated patient shows a period of reduced background EMG, lasting about $14 \mathrm{~ms}$, after the cMEP response before the suppression fully develops at a latency of $40 \mathrm{~ms}$. In seven of the nine treated patients such a period of weaker suppression of voluntary EMG was identifiable before a stronger suppression developed.

The mean (SEM) latency of maximum suppression of EMG during the silent period was longer (unpaired $t$ test; $\mathrm{P}<0.01$ ) in the patients taking antipsychotic medication (38.7 (2.4) $\mathrm{ms})$ than in the drug naïve patients $(28.2(0.7)$ $\mathrm{ms})$. This suggests that the inhibitory processes producing the early part of the silent period seen in drug naïve patients may have been influenced by the medication. Figure $3 \mathrm{~B}$ shows a bar chart comparing latency of onset of maximum suppression in drug naïve and treated patients.

The mean (SEM) total duration of the silent period was no different (unpaired $t$ test; $\mathrm{P}>0.05)$ in the medicated patients (29 (2.9) $\mathrm{ms}$ ) and the drug naïve patients $(27.3(2.9) \mathrm{ms})$.

The silent period was clearly divided into an early component (early SP) with weaker suppression of voluntary EMG and a later
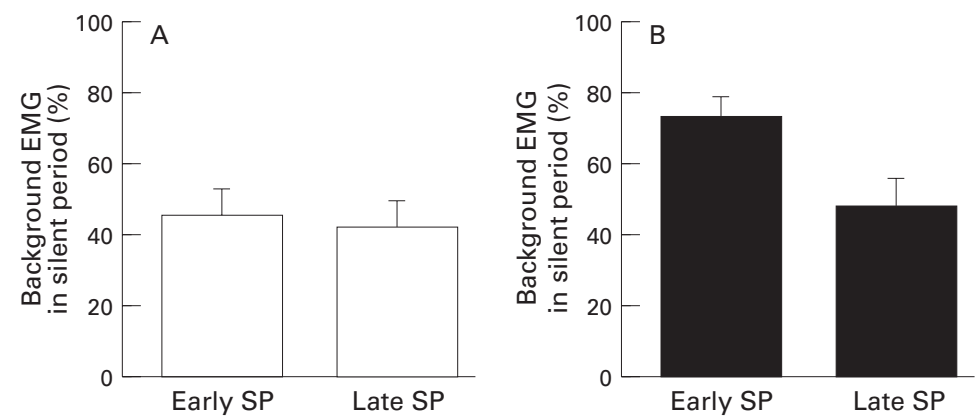

Figure 4 Percentage of voluntary background EMG present during the early and late portions of the silent period $(A)$ in drug naive patients and $(B)$ in medicated patients. There is a higher $(P<0.05)$ percentage of background EMG present during the early SP in the medicated patients than in the late SP. Error bars represent one SEM. component (late SP) in seven of the nine patients taking medication. In the remaining two medicated patients and in the nine drug naïve patients, there was no such divisions in the silent period. In these patients, the silent period was divided into two equal halves (see methods). Figure 4 shows the percentage of background EMG present during the early and late portions of the silent period in the two groups.

The mean (SEM) level of background EMG present in drug naïve patients (figure 4A) was $54.7 \%(7.3) \%$ of control levels during the early SP and $58 \%(7.8 \%)$ of control levels during the late SP. There was no significant difference between the reduction of background EMG during the two parts of the silent period in the drug naïve patients (paired $t$ test; $\mathrm{P}>0.05$ ). Figure $4 \mathrm{~B}$ shows the percentage of background EMG present in the early and late components of the silent period in the medicated patients. The mean level of EMG present was significantly greater (paired $t$ test; $\mathrm{P}<0.05)$ during the early SP $(73.9$ (5.5)\% of control levels) than during the late SP (48.2 (7.7)\% of control levels). The percentage of background EMG present during the early SP of the treated patients was also significantly greater (Bonferroni $t$ test; $\mathrm{P}<0.01$ ) than during either component of the SP seen in the drug naïve patients.

\section{Discussion}

The patients taking antipsychotic medication showed no differences from drug naïve patients in the latency of cMEP responses to the TMS, threshold stimulus required to evoke cMEPs, or total duration of the silent period. However, when the silent period after the cMEP response was examined, the patients taking medication exhibited an early component of weaker suppression of EMG before a later stronger period of suppression developed. This finding was reflected by a longer latency of maximum suppression of voluntary EMG in the patients established on antipsychotic medication than in drug naïve patients.

There was no difference in the positive (SAPS) and negative (SANS) symptom scores in medicated and drug naïve patients. Neither was there any difference in the duration of 
illness in the two groups. This might be of importance as some assessments made in schizophrenia such as latent inhibition, ${ }^{16}$ are known to be more severely affected during the acute stage of the illness. These findings increased our confidence that the differences in corticospinal physiology between these two groups of patients were due to the medication rather than the clinical characteristics of the illness.

INTRACORTICAL INHIBITORY MECHANISM

The suppression of EMG activity in response to TMS is produced, at least in part, by activation of inhibitory interneurons within the cortex reducing cortical output through pyramidal pathways. ${ }^{717}$ The fact that suppression of EMG can sometimes be seen in the absence of cMEPs suggests that TMS can activate these putative inhibitory interneurons more readily than excitatory cortical pathways. ${ }^{7}$ This could occur if the structures excited by TMS and responsible for suppression were in closer proximity to the stimulating coil. Jones ${ }^{18}$ reported that inhibitory GABAergic neurons are located throughout the primate motor cortex, but they predominate in the most superficial layers I and II. The more superficial inhibitory interneurons, despite their smaller axon diameter, ${ }^{18}$ might therefore be activated preferentially by TMS of lower stimulus strength as a consequence of the shorter distance between their processes and the stimulating coil. Although the antipsychotic medication used in schizophrenia is known to have a broad range of activity within the CNS, its major known influence is on the dopaminergic system (see below). It seems more likely that the increased latency of maximum suppression of EMG found after medication results from disruption to dopaminergic systems within the basal ganglia than a direct effect on GABAergic systems within the cortex itself. It may be that inputs from the basal ganglia to the inhibitory circuitry of the motor cortex might be disrupted as a result of the medication and so alter the strength of inhibition during the early portion of the silent period.

EFFECT OF NEUROLEPTIC MEDICATION ON THE DOPAMINE SYSTEM

The antipsychotic medication given to the drug naïve patients in this study binds to dopamine receptors. ${ }^{23}$ Indeed, when used at clinical doses, such medication can induce parkinsonian symptoms such as rigidity and bradykinesia that can only be reversed by removal of the drug or addition of antiparkinsonian agents (see for example, Fielding and $\mathrm{Lal}^{19}$ ). It is well known that antipsychotic drugs have a delayed action on blocking presynaptic dopamine activity and that this closely mirrors the time course of their antipsychotic efficacy. ${ }^{4}$ Our next objective is to examine when, with respect to the onset of medication, alteration in the form of the of suppression of EMG during the silent period occurs. Furthermore, it is possible that this information will provide clinicians with a useful physiological marker of antipsychotic function.
PARKINSON'S DISEASE AND SUPPRESSION OF EMG TO TMS

Our present study has shown that dopaminergic antagonists, given as neuroleptic drugs, can increase the latency of maximal suppression of voluntary EMG in the silent period. It is therefore relevant to compare our findings with studies investigating the silent period in Parkinson's disease, in which dopamine is pathologically depleted. Haug et $a l^{20}$ reported a shorter silent period in Parkinson's disease. Priori et $a l^{1}$ reported similar reductions in the duration of the silent periods in both idiopathic and drug induced (with neuroleptic drugs) parkinsonism. In both studies, levodopa therapy restored the duration of the silent period to normal, perhaps reflecting restoration of function of dopaminergic inhibitory circuitry.

In the current study we have seen that maximum suppression of EMG takes longer to develop during the silent period. This probably reflects a weakening of suppression at a time equivalent to the early part of the silent period. Ridding $e t a l^{2}$ have found a reduction in the strength of inhibition in the early part of the silent period in Parkinson's disease using paired TMS pulses delivered with interstimulus intervals of $1-5 \mathrm{~ms}$. Their result is consistent with our finding that voluntary contraction is suppressed less effectively during the early part of the silent period in patients taking antidopaminergic medication.

\section{Conclusion}

In conclusion, it is clear that a disruption of dopaminergic systems as a result of antipsychotic medication can affect the silent period of suppressed EMG in response to TMS during a voluntary contraction as has been seen in Parkinson's disease. The present study has shown that changes in the latency of maximal suppression can occur in schizophrenic patients who have been established on neuroleptic medication. Our future objectives will be to investigate the time course of this change and to look at the possibility of using the motor response to TMS as a marker for the status of antipsychotic medication.

We thank all the patients who participated in this study.

1 Carlsson A, Lindquist M. Effect of chlorpromazine or haloperidol on the formation of 3-methoxytyramine and normetanephrine in mouse brain. Acta Pharmacol Toxicol 1963;20:140-4.

2 Seeman P, Lee T, Chau-Wong M, Wong K. Antipsychotic drug doses and neuroleptic/dopamine receptors. Nature 1976;261:717-9.

3 Creese I, Burt DR, Snyder SH. Dopamine receptor binding predicts clinical and pharmacologic potencies of antischizophrenic drugs. Science 1976;192:481-3.

4 Pickar D, Labarca R, Linnoila M, Roy A, Hommer D, Everett D, Paul SM. Neuroleptic-induced decrease in plasma homovanillic acid and antipsychotic activity in schizophrenic patients. Science 1984;225:954-7.

5 Hollister LE. New psychotherapeutic drugs. f Clin Psychopharm 1994;14:50-63.

6 Barker AT, Jalinous R, Freeston IL. Non-invasive magnetic stimulation of human motor cortex [letter]. Lancet 1985;i: 1106-7.

7 Davey NJ, Romaiguère P, Maskill DW, Ellaway $\mathrm{PH}$. Suppression of voluntary motor activity revealed using transcranial magnetic stimulation of the motor cortex in transcranial magnetic stimulation

8 Rothwell JC, Thompson PD, Day BL, Boyd S, Marsden CD. Stimulation of the human motor cortex through the scalp. Exp Physiol 1991;76:159-200. 
9 Puri BK, Davey NJ, Ellaway PH, Lewis SW. An investigation of motor function in schizophrenia using transcranial magnetic stimulation of the motor cortex. Br f Psychiatry 1996 169:690-5.

10 Davey NJ, Puri BK, Lewis HS, Lewis SW, Ellaway PH. Electromyographic resonses of thenar muscles to transcrnial magnetic stimulation of the motor cortex in schizophrenic patients. F Physiol 1995;489:31P.

11 Puri BK, Davey NJ, Lewis SW. The effects of antipsychotic medication on electromyographic responses to transcranial magnetic stimulation of the motor cortex in schizophrenic patients. Schizophrenia Res 1996;18:234.

12 American Psychiatric Association. Diagnostic and statistical manual of mental disorders, 4th ed. Washington, DC: American Psychiatric Association, 1994.

13 Guy W. The clinical global impression scale (CGI). In: Guy W, ed. Assessment manual for psychopharmocology. Washington, DC: US Government Printing Office, 1976. (DHEW ton, DC: US Government

14 Day BL, Dressler D, Hess CW, Maertens De Noordhout A Day BL, Dressler D, Hess CW, Maertens De Noordhout A,
Marsden CD, Mills K, et al. Direction of current in magnetic stimulating coils used for percutaneous activation of brain, spinal cord and peripheral nerve. I Physiol of brain, spin

15 Ellaway PH. Cumulative sum technique and its application to the analysis of peristimulus time histograms. Electroen cephalogr Clin Neurophysiol 1978;45:302-4.
16 Baruch I, Hemsley DR, Gray JA. Differential performance of acute and chronic schizophrenics in a latent inhibition of acute and chronic schizophrenics in a
task. $\mathcal{F}$ Nerv Ment Dis $1988 ; \mathbf{1 7 6} 598-606$.

17 Ferbert A, Priori A, Rothwell JC, Day BL, Colebatch JG, Marsden CD. Interhemispheric inhibition of the human motor cortex. F Physiol 1992;453:525-46.

18 Jones EG. Varieties and distribution of non-pyramidal cells in the somatic sensory cortex of the squirrel monkey. $\mathcal{F}$ Comp Neurol 1975;160:205-67.

19 Fielding S, Lal H. Behavioural actions of neuroleptics. In: Iversen LL, Iversen SD, Snyder SH, eds. Handbook of psychopharmacology. Vol 10. New York: Plenum Press, 1978:91-128

20 Haug BA, Schonle PW, Knobloch C, Kohne M. Silent period measurement revives as a valuable diagnostic tool with transcranial magnetic stimulation. Electroencephalogr Clin Neurophysiol 1992;85:158-60.

21 Priori A, Berardelli A, Inghilleri M, Accornero N, Manfredi M. Motor cortical inhibition and the dopaminergic system. Pharmacological changes in the silent period after transcranial brain stimulation in normal subjects, patients with Parkinson's disease and drug-induced parkinsonism. Brain 1994;117:317-23.

22 Ridding MC, Inzelberg R, Rothwell JC. Changes in the excitability of motor cortical circuitry in patients with Parkinson's disease. Ann Neurol 1995;37:181-8. 